\title{
SMOOTHNESS CONDITIONS ON MEASURES USING WALLMAN SPACES
}

\author{
CHARLES TRAINA
}

(Received 15 October 1997 and in revised form 13 April 1998)

\begin{abstract}
In this paper, $\mathbf{X}$ denotes an arbitrary nonempty set, $\mathscr{L}$ a lattice of subsets of $\mathbf{X}$ with $\varnothing, \mathbf{X} \in \mathscr{L}, \mathbf{A}(\mathscr{L})$ is the algebra generated by $\mathscr{L}$ and $M(\mathscr{L})$ is the set of nontrivial, finite, and finitely additive measures on $\mathbf{A}(\mathscr{L})$, and $M_{R}(\mathscr{L})$ is the set of elements of $M(\mathscr{L})$ which are $\mathscr{L}$-regular. It is well known that any $\mu \in M(\mathscr{L})$ induces a finitely additive measure $\bar{\mu}$ on an associated Wallman space. Whenever $\mu \in M_{R}(\mathscr{L}), \bar{\mu}$ is countably additive.

We consider the general problem of given $\mu \in M(\mathscr{L})$, how do properties of $\bar{\mu}$ imply smoothness properties of $\mu$ ? For instance, what conditions on $\bar{\mu}$ are necessary and sufficient for $\mu$ to be $\sigma$-smooth on $\mathscr{L}$, or strongly $\sigma$-smooth on $\mathscr{L}$, or countably additive? We consider in discussing these questions either of two associated Wallman spaces.
\end{abstract}

Keywords and phrases. Wallman spaces, outer measure associated with a lattice measure, smoothness of a lattice measure, regular outer measure.

1991 Mathematics Subject Classification. 28A12, $28 \mathrm{C} 15$.

1. Introduction. We give some standard notational and background material in Section 2, related matters can be found in $[1,4,11]$. In Section 3, we answer questions of smoothness of $\mu$ in terms of outer measures (associated with $\bar{\mu}$ ) of sets in the Wallman spaces. Section 4 gives smoothness conditions for $\mu$ assuming the measurability of certain sets in the Wallman spaces.

Some related materials concerning these outer measures can be found in $[5,6,8$, $9,10]$.

2. Background and notation. We introduce the necessary measure theoretic, and lattice definitions. The definitions and notations are standard and are consistent with those found in $[1,2,3,4,11]$. We collect the one's we need for the reader's convenience.

Throughout, $\mathbf{X}$ denotes an arbitrary nonempty set, $\mathscr{L}$ a lattice of subsets of $\mathbf{X}$ such that $\varnothing, \mathbf{X} \in \mathscr{L}$. For $E \subset \mathbf{X}, E^{\prime}=\mathbf{X}-E$ denotes its complement. We denote by $\mathbf{A}(\mathscr{L})$, the algebra generated by $\mathscr{L} ; \delta(\mathscr{L})$, the lattice of all countable intersections of sets from $\mathscr{L} ; \tau(\mathscr{L})$, the lattice of arbitrary intersections of sets from $\mathscr{L} ; \mathscr{L}^{\prime}$, the lattice of complements of sets from $\mathscr{L}$. The necessary measure theoretic definitions follow.

The set of all nonnegative finite valued, nontrivial, finitely additive, and bounded measures on $\mathbf{A}(\mathscr{L})$ is denoted by $M(\mathscr{L})$.

An element $\mu \in M(\mathscr{L})$ is said to be $\sigma$-smooth on $\mathscr{L}$ if and only if whenever $L_{n} \in \mathscr{L}$, $n=1,2, \ldots$, and $L_{n} \downarrow \varnothing, \mu\left(L_{n}\right) \rightarrow 0$.

An element $\mu \in M(\mathscr{L})$ is said to be $\sigma$-smooth on $\mathbf{A}(\mathscr{L})$ if and only if whenever $A_{n} \in$ $\mathrm{A}(\mathscr{L}), n=1,2, \ldots$, and $A_{n} \downarrow \varnothing, \mu\left(A_{n}\right) \rightarrow 0$. (We note that this condition is equivalent 
to $\mu$ being countably additive.)

An element $\mu \in M(\mathscr{L})$ is said to be strongly $\sigma$-smooth on $\mathscr{L}$ if and only if whenever $L, L_{n} \in \mathscr{L}, n=1,2, \ldots$; and $L_{n} \downarrow L, L=\bigcap_{n=1}^{\infty} L_{n}, \mu(L)=\inf \left\{\mu\left(L_{n}\right) \mid n=1,2, \ldots\right\}$.

An element $\mu \in M(\mathscr{L})$ is said to be $\mathscr{L}$-regular if and only if, for any $A \in \mathbf{A}(\mathscr{L})$, $\mu(A)=\sup \{\mu(L) \mid L \subset A, L \in \mathscr{L}\}$.

The following notations is used to denote the subsets of $M(\mathscr{L})$ determined by the preceding properties

(a) $M_{\sigma}(\mathscr{L})$ is the set of measures that are $\sigma$-smooth on $\mathscr{L}$;

(b) $M^{\sigma}(\mathscr{L})$ is the set of measures that are $\sigma$-smooth on $\mathbf{A}(\mathscr{L})$;

(c) $J(\mathscr{L})$ is the set of measures that are strongly $\sigma$-smooth on $\mathscr{L}$;

(d) $M_{R}(\mathscr{L})$ is the set of $\mathscr{L}$-regular measures;

(e) $M_{R}^{\sigma}(\mathscr{L})$ is the set of $\mathscr{L}$-regular measures of $M^{\sigma}(\mathscr{L})$.

We note that $M^{\sigma}(\mathscr{L}) \subset M_{\sigma}(\mathscr{L})$, and $M_{R}(\mathscr{L}) \cap M_{\sigma}(\mathscr{L}) \subset M^{\sigma}(\mathscr{L})$. We denote by $I(\mathscr{L})$, $I_{\sigma}(\mathscr{L}), I^{\sigma}(\mathscr{L}), \Phi(\mathscr{L}), I_{R}(\mathscr{L})$, and $I_{R}^{\sigma}(\mathscr{L})$ the subsets of the corresponding $M$ 's that consist of the nontrivial $0-1$ valued measures.

We write $\mu \leq v(\mathscr{L})$ whenever $\mu, v$ are measures, or set functions such that $\mu(L) \leq$ $v(L)$ for all $L \in \mathscr{L}$.

We now recall some lattice definitions

(a) A lattice $\mathscr{L}$ is said to be $T_{0}$ if and only if, given any two distinct points of $\mathbf{X}$, there is a lattice set containing one and not the other.

(b) A lattice $\mathscr{L}$ is said to be disjunctive if and only if, for any $x \in \mathbf{X}$ and $A \in \mathscr{L}$ such that $x \notin A$, there exists an $L \in \mathscr{L}$ such that $x \in L, A \cap L=\varnothing$.

(c) A lattice $\mathscr{L}$ is normal if, for any $A, B \in \mathscr{L}$ with $A \cap B=\varnothing$, there exists $L, K \in \mathscr{L}$ with $A \subset L^{\prime}, B \subset K^{\prime}$, and $L^{\prime} \cap K^{\prime}=\varnothing$.

(d) A lattice $\mathscr{L}$ is a delta lattice ( $\delta$-lattice) if $\delta(\mathscr{L})=\mathscr{L}$.

(e) A lattice $\mathscr{L}$ is compact if, for any collection $\left\{L_{\alpha}\right\}$ of sets from $\mathscr{L}, \bigcap_{\alpha} L_{\alpha}=\varnothing$ implies that there exists $L_{\alpha_{1}}, \ldots, L_{\alpha_{n}}$ with $\bigcap_{i=1}^{n} L_{\alpha_{i}}=\varnothing$.

(f) A lattice $\mathscr{L}$ is countably compact if and only if, for every sequence $\left\{L_{n}\right\}$ from $\mathscr{L}$ with $\bigcap_{n=1}^{\infty} L_{n}=\varnothing$, there exists $L_{n_{1}}, \ldots, L_{n_{k}}$ with $\bigcap_{i=1}^{k} L_{n_{i}}=\varnothing$.

(g) If $\mathscr{L}_{1}$ and $\mathscr{L}_{2}$ are two lattices of subsets of $\mathrm{X}$, then $\mathscr{L}_{1}$ separates $\mathscr{L}_{2}$ if and only if, whenever $A, B \in \mathscr{L}_{2}$ with $A \cap B=\varnothing$ implies that there exists $C, D \in \mathscr{L}_{1}$ with $A \subset C$, $B \subset D$, and $C \cap D=\varnothing$.

For $\mu \in M(\mathscr{L})$, the support of $\mu$ is the set

$$
S(\mu)=\{L \in \mathscr{L} \mid \mu(L)=\mu(\mathbf{X})\} .
$$

We briefly recall two of the generalized Wallman spaces that will play a central role in this paper. For further details, see [1, 2, 3, 11].

If $\mathscr{L}$ is $T_{0}$ and disjunctive we define, for $A \in \mathbf{A}(\mathscr{L}), W(A)=\left\{\mu \in I_{R}(\mathscr{L}) \mid \mu(A)=1\right\}$.

Clearly, for $A, B \in \mathbf{A}(\mathscr{L})$,

(1) $W(A \cup B)=W(A) \cup W(B)$,

(2) $W(A \cap B)=W(A) \cap W(B)$,

(3) $W\left(A^{\prime}\right)=W(A)^{\prime}$,

(4) $A \subset B$ if and only if $W(A) \subset W(B)$,

(5) $W(\mathbf{A}(\mathscr{L}))=\mathbf{A}(W(\mathscr{L}))$. 
$W(\mathscr{L})=\{W(L) \mid L \in \mathscr{L}\}$ is taken as a base for the closed sets of a topology $\tau W(\mathscr{L})$. $W(\mathscr{L})$ is a compact lattice and, therefore, $\tau W(\mathscr{L})$ is compact and $W(\mathscr{L})$ separates $\tau W(\mathscr{L}) . I_{R}(\mathscr{L}), \tau W(\mathscr{L})$ is a compact $T_{1}$ space and is $T_{2}$ if and only if $\mathscr{L}$ is normal. In addition $\mathrm{X}$ is embedded in $I_{R}(\mathscr{L})$ by the mapping

$$
\begin{aligned}
& X \longrightarrow I_{R}(\mathscr{L}), \\
& x \longrightarrow \mu_{x},
\end{aligned}
$$

where $\mu_{x}$ is the Dirac measure concentrated at $x \in \mathbf{X}$, i.e.,

$$
\mu_{x}(A)=\left\{\begin{array}{ll}
1, & \text { if } x \in A, \\
0, & \text { if } x \notin A,
\end{array} \quad A \in \mathbf{A}(\mathscr{L}) .\right.
$$

As known, specific choices of $\mathbf{X}$ and $\mathscr{L}$ yield well-known compactifications of $\mathbf{X}$.

Similarly, if $\mathscr{L}$ is just $T_{0}$, we consider the second Wallman space: for $A \in \mathbf{A}(\mathscr{L})$, let $V(A)=\{\mu \in I(\mathscr{L}) \mid \mu(A)=1\}$.

Properties (1)-(5) hold with $V$ in place of $W$, and taking $V(\mathscr{L})$ as a base for the closed sets, we get the space $I(\mathscr{L}), \tau V(\mathscr{L})$ which is compact, $T_{0}$. Clearly, $\mathbf{X}$ is embedded in $I(\mathscr{L})$ by

$$
\begin{aligned}
& X \rightarrow I(\mathscr{L}), \\
& x \longrightarrow \mu_{x} .
\end{aligned}
$$

Next, let $\mu \in M(\mathscr{L})$ and define for $A \in \mathbf{A}(\mathscr{L})$,

$$
\bar{\mu}(W(A))=\mu(A) .
$$

It is easy to see that $\bar{\mu} \in M(W(\mathscr{L}))$, and that the mapping $\mu \rightarrow \bar{\mu}$ is a bijection from $M(\mathscr{L})$ to $M(W(\mathscr{L}))$. Also, since $W(\mathscr{L})$ is a compact space, $M(W(\mathscr{L}))=M_{\sigma}(W(\mathscr{L}))$. If $\mu \in M_{R}(\mathscr{L})$, then $\bar{\mu} \in M_{R}(W(\mathscr{L}))=M_{R}^{\sigma}(W(\mathscr{L}))$. Thus $\bar{\mu}$, in this case, can be extended to $\sigma\left(W(\mathscr{L})\right.$ ) even to $S_{\bar{\mu}^{*}}$ and is $\delta W(\mathscr{L})$ regular on these sets. We recall the following theorem from [2].

THEOREM 2.1. Let $\mathscr{L}$ be a lattice of subsets of the nonempty set $\mathbf{X}$, where $\mathscr{L}$ is $T_{0}$ and disjunctive. The following are equivalent

(1) $\mu \in M_{R}^{\sigma}(\mathscr{L})$;

(2) $\bar{\mu}\left(\bigcap_{i=1}^{\infty} W\left(L_{i}\right)\right)=0, \bigcap_{i=1}^{\infty} W\left(L_{i}\right) \subset I_{R}(\mathscr{L})-\mathbf{X}, L_{i} \downarrow, L_{i} \in \mathscr{L}$;

(3) $\bar{\mu}\left(\bigcap_{i=1}^{\infty} W\left(L_{i}\right)\right)=0, \bigcap_{i=1}^{\infty} W\left(L_{i}\right) \subset I_{R}(\mathscr{L})-I_{R}^{\sigma}(\mathscr{L}), L_{i} \downarrow, L_{i} \in \mathscr{L}$;

(4) $\bar{\mu}^{*}(\mathbf{X})=\bar{\mu}\left(I_{R}(\mathscr{L})\right)$.

One of our main aims is to extend this theorem.

In a similar manner, for $\mu \in M(\mathscr{L})$ and $A \in \mathbf{A}(\mathscr{L})$, we define $\bar{\mu}(V(A))=\mu(A)$. Again, $\mu \rightarrow \bar{\mu}$ is a bijection of $M(\mathscr{L})$ to $M(V(\mathscr{L}))$ and $M(V(\mathscr{L}))=M_{\sigma}(V(\mathscr{L}))$.

In either of the two Wallman spaces, certain smoothness properties of $\mu \in M(\mathscr{L})$ are directly reflected to $\bar{\mu}$. For example, $\mu \in J(\mathscr{L})\left(M^{\sigma}(\mathscr{L})\right)$ implies that $\bar{\mu} \in J(W(\mathscr{L}))\left(M^{\sigma}(W\right.$ $(\mathscr{L}))$ and similarly for $V(\mathscr{L})$. What we seek first in this paper is an analogue to Theorem 2.1 without the regularity assumptions for smoothness. To elaborate, if $\mu \in M(\mathscr{L})$, then $\bar{\mu}$ always belongs to $M_{\sigma}(W(\mathscr{L}))\left(M_{\sigma}(V(\mathscr{L}))\right)$ and to obtain necessary 
and sufficient conditions for a $\mu \in M(\mathscr{L})$ to be in $M_{\sigma}(\mathscr{L})$ in terms of $\bar{\mu}$, we must look elsewhere than just for smoothness on $W(\mathscr{L})(V(\mathscr{L}))$.

We recall that, for a $\mu \in M(\mathscr{L})$, there are several associated outer measures (see $[6,8,9,10])$ which are defined as follows: for any $E \subset \mathbf{X}$,

$$
\mu^{\prime}(E)=\inf \left\{\mu\left(L^{\prime}\right) \mid E \subset L^{\prime}, L \in \mathscr{L}\right\} ;
$$

$\mu^{\prime}$ is just finitely subadditive;

$$
\mu^{\prime \prime}(E)=\inf \left\{\sum_{i=1}^{\infty} \mu\left(L_{i}^{\prime}\right) \mid E \subset \bigcup_{i=1}^{\infty} L_{i}^{\prime}, L_{i} \in \mathscr{L}\right\}
$$

and

$$
\mu^{*}(E)=\inf \left\{\sum_{i=1}^{\infty} \mu\left(A_{i}\right) \mid E \subset \bigcup_{i=1}^{\infty} A_{i}, A_{i} \in \mathbf{A}(\mathscr{L})\right\} .
$$

The outer measures $\mu^{\prime \prime}$ and $\mu^{*}$ are both countably subadditive.

Finally,

$$
\mu^{\bullet}(E)=\inf \{\mu(A) \mid E \subset A, A \in \mathbf{A}(\mathscr{L})\},
$$

which is only finitely subadditive.

We have the following relations between $\mu$ and these outer measures. $\mu^{\bullet}=\mu$ on $\mathbf{A}(\mathscr{L}), \mu^{*} \leq \mu$ on $\mathbf{A}(\mathscr{L})$. If, however, $\mu \in M^{\sigma}(\mathscr{L})$, then $\mu^{*}=\mu$ on $\mathbf{A}(\mathscr{L})$. It is clear that $\mu^{\prime}=\mu$ on $\mathscr{L}^{\prime}$; while $\mu \leq \mu^{\prime}$ on $\mathscr{L}$ and $\mu=\mu^{\prime}$ on $\mathscr{L}$ if and only if $\mu \in M_{R}(\mathscr{L})$. If $\mu \in M_{\sigma}(\mathscr{L})$, then $\mu^{\prime \prime}(\mathbf{X})=\mu(\mathbf{X})$ and $\mu \leq \mu^{\prime \prime}$ on $\mathscr{L}$ (see [6, 10]). There are other obvious relationships that hold between these outer measures, however, we do not explicitly list them.

We next consider a finite outer measure $v$ defined on all subsets of $\boldsymbol{X}$, which may be either finitely or countably subadditive.

$$
S_{v}=\left\{E \subset \mathbf{X} \mid v(G)=v(G \cap E)+\left(G \cap E^{\prime}\right) \text { for all } G \subset \mathbf{X}\right\}
$$

is the set of $v$-measurable sets.

The outer measure $v$ is regular if, for every $E \subset \mathbf{X}$, there exists an $M \in S_{v}$ such that $E \subset M$ and $\nu(E)=v(M)$. If $v$ is regular, then $E \in S_{v}$ if and only if $v(\mathbf{X})=v(E)+v\left(E^{\prime}\right)$. If $v$ is a regular countably subadditive outer measure and if $\left\{E_{n}\right\}$ is a sequence of sets from $X$ such that $E_{n} \subset E_{n+1}, n=1,2, \ldots$, then $v\left(\lim _{n \rightarrow \infty} E_{n}\right)=\lim _{n \rightarrow \infty} v\left(E_{n}\right)$. (See [7]).

The following result is known (see $[6,10])$.

THEOREM 2.2. Let $\mu \in M_{\sigma}(\mathscr{L})$. Then $\mu^{\prime}=\mu^{\prime \prime}$ on $\mathscr{L}$ if

(a) $\mathscr{L}$ is countably compact;

(b) $\mathscr{L}$ is $\delta$-normal;

(c) $\mathscr{L}$ is normal and countably paracompact.

Corollary 2.2.1. Let $\mu \in M(\mathscr{L})$, where $\mathscr{L}$ is $T_{0}$ and disjunctive, and let $\bar{\mu} \in$ $M(W(\mathscr{L}))=M_{\sigma}(W(\mathscr{L}))$ be the induced measure. Then $\bar{\mu}^{\prime}=\bar{\mu}^{\prime \prime}$ on $W(\mathscr{L})$.

REMARK. A similar result holds for $\mu \in M(\mathscr{L})$ with $\mathscr{L}$ just $T_{0}$, namely, if $\bar{\mu} \in$ $M(V(\mathscr{L}))=M_{\sigma}(V(\mathscr{L}))$, then $\bar{\mu}^{\prime}=\bar{\mu}^{\prime \prime}$ on $V(\mathscr{L})$. 
A number of these equalities can be extended. For instance, the following is known.

THEOREM 2.3. Let $\mathscr{L}_{1}, \mathscr{L}_{2}$ be lattices of subsets of $\mathrm{X}$ such that $\mathscr{L}_{1} \subset \mathscr{L}_{2}$ and $\delta\left(\mathscr{L}_{1}\right) \subset$ $\mathscr{L}_{2}$. Let $\mu \in M_{\sigma}\left(\mathscr{L}_{1}\right)$. Then if $\mathscr{L}_{1}$ separates $\mathscr{L}_{2}, \mu^{\prime}=\mu^{\prime \prime}$ on $\mathscr{L}_{1}$ implies that $\mu^{\prime}=\mu^{\prime \prime}$ on $\mathscr{L}_{2}$.

COROLLARY 2.3.1. If $\mu \in M(\mathscr{L})$ and if

(a) $\mathscr{L}$ is $T_{0}$ and disjunctive, then $\bar{\mu}^{\prime \prime}=\bar{\mu}^{\prime}$ on $\tau W(\mathscr{L})$.

(b) $\mathscr{L}$ is just $T_{0}$, then $\bar{\mu}^{\prime \prime}=\bar{\mu}^{\prime}$ on $\tau V(\mathscr{L})$.

To make our statements concise, we note that in dealing with the Wallman space $I_{R}(\mathscr{L}), \tau W(\mathscr{L})$, we always assume that $\mathscr{L}$ is $T_{0}$ and disjunctive; while in working with the Wallman space $I(\mathscr{L}), \tau V(\mathscr{L})$, we always assume that $\mathscr{L}$ is $T_{0}$. In either case, for $E \subset \mathbf{X}$, we can view (via the mapping $x \rightarrow \mu_{x}$ noted earlier) $E$ embedded in $I_{R}(\mathscr{L})$ or $I(\mathscr{L})$.

We now note some relationships among $\mu^{\prime}, \mu^{\prime \prime}$, and the induced outer measures $\bar{\mu}^{\prime}, \bar{\mu}^{\prime \prime}$.

THEOREM 2.4. Let $\mu \in M(\mathscr{L})$ and let $E \subset \mathrm{X}$. Then

(a) $\mu^{\prime}(E)=\bar{\mu}^{\prime}(E)$,

(b) $\mu^{\prime \prime}(E)=\bar{\mu}^{\prime \prime}(E)$,

(c) $\mu^{*}(E)=\bar{\mu}^{*}(E)$,

(d) $\mu^{\bullet}(E)=\bar{\mu} \cdot(E)$,

(e) for $L \in \mathscr{L}$,

$$
\begin{aligned}
& \mu^{\prime \prime}(L)=\bar{\mu}^{\prime \prime}(L) \leq \bar{\mu}^{\prime \prime}(W(L))=\bar{\mu}^{\prime}(W(L))=\mu^{\prime}(L), \\
& \mu^{\prime \prime}(L)=\bar{\mu}^{\prime \prime}(L) \leq \bar{\mu}^{\prime \prime}(V(L))=\bar{\mu}^{\prime}(V(L))=\mu^{\prime}(L) .
\end{aligned}
$$

In (a)-(e), $\bar{\mu} \in M(W(\mathscr{L}))$ or $\bar{\mu} \in M(V(\mathscr{L}))$ depending on which Wallman space we are considering.

Finally, we note the following theorem:

THEOREM 2.5. (a) $\mu \in M_{R}(\mathscr{L})$ if and only if $\bar{\mu} \in M_{R}(W(\mathscr{L})$ ).

(b) $\mu \in J(\mathscr{L})$ implies that $\bar{\mu} \in J(W(\mathscr{L}))$.

(c) $\mu \in M^{\sigma}(\mathscr{L})$ implies that $\bar{\mu} \in M^{\sigma}(W(\mathscr{L})$ ).

The corresponding results are true with $V(\mathscr{L})$ in place of $W(\mathscr{L})$.

3. Characterizations of measures in the Wallman spaces. In this section, we wish to extend the basic Theorem 2.1 to measures in $M_{\sigma}(\mathscr{L}), J(\mathscr{L})$, and $M^{\sigma}(\mathscr{L})$. In other words, we wish to characterize these measures in terms of their induced measures on the two Wallman spaces that we have considered. The proofs for the two spaces are virtually the same so we present only one of them. We begin with $M_{\sigma}(\mathscr{L})$.

THEOREM 3.1. Let $\mathscr{L}$ be a $T_{0}$ and disjunctive lattice, and for $\mu \in M(\mathscr{L}), \bar{\mu} \in M(W(\mathscr{L}))$ denotes its induced measure in the Wallman space $I_{R}(\mathscr{L}), \tau W(\mathscr{L})$.

(a) If $\mu \in M_{\sigma}(\mathscr{L})$, then $\bar{\mu}^{\prime \prime}(\mathbf{X})=\bar{\mu}\left(I_{R}(\mathscr{L})\right.$ ).

(b) If $\mu \in M(\mathscr{L})$ and if $\bar{\mu}^{\prime \prime}(\mathbf{X})=\bar{\mu}\left(I_{R}(\mathscr{L})\right)$ and if $\bar{\mu}^{\prime \prime}$ is a regular outer measure, then $\mu \in M_{\sigma}(\mathscr{L})$.

(c) If $\mu \in I(\mathscr{L})$, then $\mu \in I_{\sigma}(\mathscr{L})$ if and only if $\bar{\mu}^{\prime \prime}(\mathbf{X})=\bar{\mu}\left(I_{R}(\mathscr{L})\right)$. 
Proof. (a) Let $\mu \in M_{\sigma}(\mathscr{L})$. Since $W(\mathscr{L})$ is compact, $\bar{\mu} \in M_{\sigma}(W(\mathscr{L}))$. Then $\mu^{\prime \prime}(\mathbf{X})=$ $\bar{\mu}^{\prime \prime}(\mathbf{X})$ by Theorem 2.4. Since $\mu \in M_{\sigma}(\mathscr{L}), \mu^{\prime \prime}(\mathbf{X})=\mu(\mathbf{X})$. The definition of $\bar{\mu}$ gives $\bar{\mu}(W(\mathbf{X}))=\mu(\mathbf{X})$, but $W(\mathbf{X})=I_{R}(\mathscr{L})$. Thus, $\bar{\mu}^{\prime \prime}(\mathbf{X})=\bar{\mu}\left(I_{R}(\mathscr{L})\right)$.

(b) Let $\mu \in M(\mathscr{L})$ so that $\bar{\mu} \in M(W(\mathscr{L}))=M_{\sigma}(W(\mathscr{L}))$ (since $W(\mathscr{L})$ is compact). Let $L_{n} \in \mathscr{L}, n=1,2, \ldots, L_{n} \downarrow \varnothing, \bigcap_{n=1}^{\infty} L_{n}=\varnothing$. Then in $I_{R}(\mathscr{L}), W\left(L_{n}\right) \downarrow$ and $\bigcap_{n=1}^{\infty} W\left(L_{n}\right) \subset$ $I_{R}(\mathscr{L})-\mathbf{X}$.

Therefore, $\bigcup_{n=1}^{\infty} W\left(L_{n}^{\prime}\right) \supset \mathbf{X}$ in $I_{R}(\mathscr{L})$. By monotonicity of $\bar{\mu}^{\prime \prime}$, we have since $\mathbf{X} \subset$ $\bigcup_{n=1}^{\infty} W\left(L_{n}^{\prime}\right) \subset I_{R}(\mathscr{L})$ that

$$
\bar{\mu}^{\prime \prime}(\mathbf{X}) \leq \bar{\mu}^{\prime \prime}\left(\bigcup_{n=1}^{\infty} W\left(L_{n}^{\prime}\right)\right) \leq \bar{\mu}^{\prime \prime}\left(I_{R}(\mathscr{L})\right) .
$$

Since $\bar{\mu} \in M_{\sigma}(W(\mathscr{L}))$,

$$
\bar{\mu}^{\prime \prime}\left(I_{R}(\mathscr{L})\right)=\bar{\mu}\left(I_{R}(\mathscr{L})\right) .
$$

By hypothesis, $\bar{\mu}^{\prime \prime}(\mathbf{X})=\bar{\mu}\left(I_{R}(\mathscr{L})\right)$. By (3.1) and (3.2),

$$
\bar{\mu}\left(I_{R}(\mathscr{L})\right)=\bar{\mu}^{\prime \prime}\left(\bigcup_{n=1}^{\infty} W\left(L_{n}^{\prime}\right)\right) .
$$

Now, $\bar{\mu}\left(I_{R}(\mathscr{L})\right)=\bar{\mu}(W(\mathbf{X}))=\mu(\mathbf{X})$. Since $\bar{\mu}^{\prime \prime}$ is regular,

$$
\bar{\mu}^{\prime \prime}\left(\bigcup_{n=1}^{\infty} W\left(L_{n}^{\prime}\right)\right)=\lim _{n \rightarrow \infty} \bar{\mu}^{\prime \prime}\left(W\left(L_{n}^{\prime}\right)\right) .
$$

But

$$
\lim _{n \rightarrow \infty} \bar{\mu}^{\prime \prime}\left(W\left(L_{n}^{\prime}\right)\right) \leq \lim _{n \rightarrow \infty} \bar{\mu}^{\prime}\left(W\left(L_{n}^{\prime}\right)\right)=\lim _{n \rightarrow \infty} \bar{\mu}\left(W\left(L_{n}^{\prime}\right)\right) .
$$

Hence,

$$
\mu(\mathbf{X}) \leq \lim _{n \rightarrow \infty} \bar{\mu}\left(W\left(L_{n}^{\prime}\right)\right)
$$

However, $\bar{\mu}\left(W\left(L_{n}^{\prime}\right)\right)=\mu\left(L_{n}^{\prime}\right)$ for all $n$. So,

$$
\mu(\mathbf{X}) \leq \lim _{n \rightarrow \infty} \mu\left(L_{n}^{\prime}\right) .
$$

In $\mathbf{X}, L_{n}^{\prime} \uparrow \mathbf{X}$, so

$$
\lim _{n \rightarrow \infty} \mu\left(L_{n}^{\prime}\right) \leq \mu(\mathbf{X}) .
$$

It follows from (3.7) and (3.8) that $\lim _{n \rightarrow \infty} \mu\left(L_{n}^{\prime}\right)=\mu(\mathbf{X})$ or $\lim _{n \rightarrow \infty} \mu\left(L_{n}\right)=0$. So, $\mu \in$ $M_{\sigma}(\mathscr{L})$.

(c) This follows immediately since any zero-one valued outer measure is regular.

REMARK 1. The theorem holds with $\mathscr{L}$ just $T_{0}$ if we replace the Wallman space $I_{R}(\mathscr{L}), \tau W(\mathscr{L})$ with the space $I(\mathscr{L}), \tau V(\mathscr{L})$.

We can strengthen the previous theorem as follows.

THEOREM 3.2. (a) Let $\mathscr{L}$ be $T_{0}$ and disjunctive and let $\mu \in M(\mathscr{L})$.

(1) If $\mu \in M_{\sigma}(\mathscr{L})$, then $\bar{\mu}^{\prime \prime}\left(I_{R}^{\sigma}(\mathscr{L})\right)=\bar{\mu}\left(I_{R}(\mathscr{L})\right)$.

(2) If $\mu \in M(\mathscr{L})$ and if $\bar{\mu}^{\prime \prime}\left(I_{R}^{\sigma}(\mathscr{L})\right)=\bar{\mu}\left(I_{R}(\mathscr{L})\right)$ and if $\bar{\mu}^{\prime \prime}$ is regular,then $\mu \in M_{\sigma}(\mathscr{L})$. 
(3) If $\mu \in I(\mathscr{L})$, then $\mu \in I_{\sigma}(\mathscr{L})$ if and only if $\bar{\mu}^{\prime \prime}\left(I_{R}^{\sigma}(\mathscr{L})\right)=\bar{\mu}\left(I_{R}(\mathscr{L})\right)$.

(b) Let $\mathscr{L}$ be $T_{0}$ and $\mu \in M(\mathscr{L})$. Then

(4) If $\mu \in M_{\sigma}(\mathscr{L})$, then $\bar{\mu}^{\prime \prime}\left(I_{\sigma}(\mathscr{L})\right)=\bar{\mu}^{\prime \prime}\left(I^{\sigma}(\mathscr{L})\right)=\bar{\mu}(I(\mathscr{L}))$.

(5) If $\mu \in M(\mathscr{L}), \bar{\mu}^{\prime \prime}\left(I_{\sigma}(\mathscr{L})\right)=\bar{\mu}(I(\mathscr{L}))$, and $\bar{\mu}^{\prime \prime}$ is regular, then $\mu \in M_{\sigma}(\mathscr{L})$.

(6) If $\mu \in I(\mathscr{L})$, then $\mu \in I_{\sigma}(\mathscr{L})$ if and only if $\bar{\mu}^{\prime \prime}\left(I_{\sigma}(\mathscr{L})\right)=\bar{\mu}(I(\mathscr{L}))$.

It is clearly just necessary to prove part of this theorem because of the similarity of cases (a) and (b) and in light of the proof of the previous theorem. We, therefore, just prove case (b), parts (4) and (5).

Proof. (4) We have $\mathbf{X} \subset I_{\sigma}(\mathscr{L}) \subset I(\mathscr{L})$, where $\mathbf{X}$ is identified with its image in $I(\mathscr{L})$. By monotonicity of $\bar{\mu}^{\prime \prime}$,

$$
\bar{\mu}^{\prime \prime}(\mathbf{X}) \leq \bar{\mu}^{\prime \prime}\left(I_{\sigma}(\mathscr{L})\right) \leq \bar{\mu}^{\prime \prime}(I(\mathscr{L})) .
$$

Since $\mu \in M_{\sigma}(\mathscr{L}), \bar{\mu}^{\prime \prime}(\mathbf{X})=\bar{\mu}(I(\mathscr{L}))$. Similarly, $\bar{\mu} \in M_{\sigma}(V(\mathscr{L}))$. So, $\bar{\mu}(I(\mathscr{L}))=\bar{\mu}^{\prime \prime}(I(\mathscr{L}))$. It follows from the preceding statements that $\bar{\mu}^{\prime \prime}\left(I_{\sigma}(\mathscr{L})\right)=\bar{\mu}(I(\mathscr{L}))$. Similarly, $\mathbf{X} \subset$ $I^{\sigma}(\mathscr{L}) \subset I_{\sigma}(\mathscr{L}) \subset I(\mathscr{L})$ and so $\bar{\mu}^{\prime \prime}(\mathbf{X}) \leq \bar{\mu}^{\prime \prime}\left(I^{\sigma}(\mathscr{L})\right) \leq \bar{\mu}^{\prime \prime}\left(I_{\sigma}(\mathscr{L})\right)$. By hypothesis, $\mu \in$ $M_{\sigma}(\mathscr{L})$. So, $\bar{\mu}^{\prime \prime}(\mathbf{X})=\bar{\mu}(I(\mathscr{L}))$ and by the preceding result, $\bar{\mu}^{\prime \prime}\left(I_{\sigma}(\mathscr{L})\right)=\bar{\mu}(I(\mathscr{L}))$. It follows from these results that $\bar{\mu}^{\prime \prime}\left(I^{\sigma}(\mathscr{L})\right)=\bar{\mu}(I(\mathscr{L}))$.

(5) Let $L_{n} \in \mathscr{L}, n=1,2, \ldots$ such that $L_{n} \downarrow \varnothing$. Then in $I(\mathscr{L}), V\left(L_{n}\right) \downarrow$, and $\operatorname{so} \bigcap_{n=1}^{\infty} V\left(L_{n}\right)$ $\subset I(\mathscr{L})-X$. If $v \in \bigcap_{n=1}^{\infty} V\left(L_{n}\right)$, then $v\left(L_{n}\right)=1$ for all $n$ and so $v\left(L_{n}\right) \rightarrow 1$. Hence, $v \notin I_{\sigma}(\mathscr{L})$. Therefore, $\bigcap_{n=1}^{\infty} V\left(L_{n}\right) \subset I(\mathscr{L})-I_{\sigma}(\mathscr{L})$, and so $I_{\sigma}(\mathscr{L}) \subset \bigcup_{n=1}^{\infty} V\left(L_{n}^{\prime}\right)$. By monotonicity of $\bar{\mu}^{\prime \prime}$, it follows from the preceding set inclusion that

$$
\bar{\mu}^{\prime \prime}\left(I_{\sigma}(\mathscr{L})\right) \leq \bar{\mu}^{\prime \prime}\left(\bigcup_{n=1}^{\infty} V\left(L_{n}^{\prime}\right)\right) \leq \bar{\mu}^{\prime \prime}(I(\mathscr{L})) .
$$

By hypothesis, $\bar{\mu}^{\prime \prime}\left(I_{\sigma}(\mathscr{L})\right)=\bar{\mu}(I(\mathscr{L}))$. Since $V(\mathscr{L})$ is compact, $\bar{\mu} \in M_{\sigma}(V(\mathscr{L}))$ and so $\bar{\mu}^{\prime \prime}(I(\mathscr{L}))=\bar{\mu}(I(\mathscr{L}))$. It follows from (3.10) and the preceding two equalities that

$$
\bar{\mu}(I(\mathscr{L}))=\bar{\mu}^{\prime \prime} \bigcup_{n=1}^{\infty}\left(V\left(L_{n}^{\prime}\right)\right) .
$$

But

$$
\bar{\mu}(I(\mathscr{L}))=\bar{\mu}(V(\mathbf{X}))=\mu(\mathbf{X})
$$

So,

$$
\mu(\mathbf{X})=\bar{\mu}^{\prime \prime}\left(\bigcup_{n=1}^{\infty} V\left(L_{n}^{\prime}\right)\right) .
$$

Since $\bar{\mu}^{\prime \prime}$ is regular,

$$
\bar{\mu}^{\prime \prime}\left(\bigcup_{n=1}^{\infty} V\left(L_{n}^{\prime}\right)\right)=\lim _{n \rightarrow \infty} \bar{\mu}^{\prime \prime}\left(V\left(L_{n}^{\prime}\right)\right) .
$$

For each $n$,

$$
\bar{\mu}^{\prime \prime}\left(V\left(L_{n}^{\prime}\right)\right) \leq \bar{\mu}^{\prime}\left(V\left(L_{n}^{\prime}\right)\right)=\bar{\mu}\left(V\left(L_{n}^{\prime}\right)\right)=\mu\left(L_{n}^{\prime}\right) .
$$

By (3.13), (3.14), and (3.15), we get

$$
\mu(X) \leq \lim _{n \rightarrow \infty} \mu\left(L_{n}^{\prime}\right) .
$$


Since $L_{n} \downarrow \varnothing, L_{n}^{\prime} \uparrow \mathbf{X}$, and so $\mu\left(L_{n}^{\prime}\right) \leq \mu(\mathbf{X})$ for all $n$. Therefore,

$$
\lim _{n \rightarrow \infty} \mu\left(L_{n}^{\prime}\right) \leq \mu(\mathbf{X}) .
$$

It follows from (3.16) and (3.17) that $\mu(\mathbf{X})=\lim _{n \rightarrow \infty} \mu\left(L_{n}^{\prime}\right)$, whence, $\lim _{n \rightarrow \infty} \mu\left(L_{n}\right)=0$ whenever $L_{n} \downarrow \varnothing$ in $\mathscr{L}$. Hence, $\mu \in M_{\sigma}(\mathscr{L})$.

We now turn our attention to the case $\mu \in M^{\sigma}(\mathscr{L})$, and we wish to obtain analogs to the previous two theorems. Again, we can consider either of the two pairs $I_{R}(\mathscr{L})$, $W(\mathscr{L})$ or $I(\mathscr{L}), V(\mathscr{L})$. We will consider the latter just for brevity.

THEOREM 3.3. Let $\mathscr{L}$ be $T_{0}$ and let $\mu \in M(\mathscr{L})$.

(1) If $\mu \in M^{\sigma}(\mathscr{L})$, then $\bar{\mu}^{*}(\mathbf{X})=\bar{\mu}^{*}\left(I_{\sigma}(\mathscr{L})\right)=\bar{\mu}^{*}\left(I^{\sigma}(\mathscr{L})\right)=\bar{\mu}(I(\mathscr{L}))$.

(2) If $\mu \in M(\mathscr{L})$ and if $\bar{\mu}^{*}$ is regular, then if either $\bar{\mu}^{*}(\mathbf{X})=\bar{\mu}(I(\mathscr{L}))$ or if $\bar{\mu}^{*}\left(I^{\sigma}(\mathscr{L})\right)=$ $\bar{\mu}(I(\mathscr{L}))$, then $\mu \in M^{\sigma}(\mathscr{L})$.

(3) If $\mu \in I(\mathscr{L})$, then $\mu \in I^{\sigma}(\mathscr{L})$ if and only if $\bar{\mu}^{*}(\mathbf{X})=\bar{\mu}(I(\mathscr{L}))$, or $\bar{\mu}^{*}\left(I^{\sigma}(\mathscr{L})\right)=$ $\bar{\mu}(I(\mathscr{L}))$.

Proof. The proof is similar to that of Theorem 3.2. We just prove (1) and part of (2).

(1) Let $\mu \in M^{\sigma}(\mathscr{L})$. In $I(\mathscr{L}), \mathbf{X} \subset I^{\sigma}(\mathscr{L}) \subset I_{\sigma}(\mathscr{L}) \subset I(\mathscr{L})$. Since $\mu \in M^{\sigma}(\mathscr{L}), \bar{\mu} \in$ $M^{\sigma}(V(\mathscr{L}))$. Thus, we have $\mu^{*}(\mathbf{X})=\mu(\mathbf{X})$ and $\bar{\mu}^{*}(I(\mathscr{L}))=\bar{\mu}(I(\mathscr{L}))$. Then

$$
\bar{\mu}(I(\mathscr{L}))=\bar{\mu}(V(\mathbf{X}))=\mu(\mathbf{X})=\mu^{*}(\mathbf{X})=\bar{\mu}^{*}(\mathbf{X}) .
$$

But,

$$
\bar{\mu}^{*}(\mathbf{X}) \leq \bar{\mu}^{*}\left(I^{\sigma}(\mathscr{L})\right) \leq \bar{\mu}^{*}\left(I_{\sigma}(\mathscr{L})\right) \leq \bar{\mu}^{*}(I(\mathscr{L}))=\bar{\mu}(I(\mathscr{L})) .
$$

Therefore,

$$
\bar{\mu}^{*}(\mathbf{X})=\bar{\mu}^{*}\left(I_{\sigma}(\mathscr{L})\right)=\bar{\mu}^{*}\left(I^{\sigma}(\mathscr{L})\right)=\bar{\mu}(I(\mathscr{L})) .
$$

(2) Let $\mu \in M(\mathscr{L})$ with $\bar{\mu}^{*}$ regular, and $\bar{\mu}^{*}\left(I^{\sigma}(\mathscr{L})\right)=\bar{\mu}(I(\mathscr{L}))$. Suppose that $A_{n} \in$ $\mathrm{A}(\mathscr{L}), n=1,2, \ldots$ with $A_{n} \downarrow \varnothing$ so that $\bigcap_{n=1}^{\infty} A_{n}=\varnothing$. Since $A_{n} \downarrow \varnothing$ in $\mathbf{X}, V\left(A_{n}\right) \downarrow$ in $I(\mathscr{L})$ so that $\bigcap_{n=1}^{\infty} V\left(A_{n}\right) \subset I(\mathscr{L})-\mathbf{X}$. If $v \in \bigcap_{n=1}^{\infty} V\left(A_{n}\right)$, then $v\left(A_{n}\right)=1$ for all $n$ and so $v\left(A_{n}\right) \rightarrow 1$, whence, $v \notin I^{\sigma}(\mathscr{L})$. Therefore, $\bigcap_{n=1}^{\infty} V\left(A_{n}\right) \subset I(\mathscr{L})-I^{\sigma}(\mathscr{L})$, and so $I^{\sigma}(\mathscr{L}) \subset \bigcup_{n=1}^{\infty} V\left(A_{n}^{\prime}\right)$. By monotonicity of $\bar{\mu}^{*}$,

$$
\bar{\mu}^{*}\left(I^{\sigma}(\mathscr{L})\right) \leq \bar{\mu}^{*}\left(\bigcup_{n=1}^{\infty} V\left(A_{n}^{\prime}\right)\right) \leq \bar{\mu}^{*}(I(\mathscr{L})) \leq \bar{\mu}(I(\mathscr{L})) .
$$

The hypothesis $\bar{\mu}^{*}\left(I^{\sigma}(\mathscr{L})\right)=\bar{\mu}(I(\mathscr{L}))$, together with the preceding inequality, yields

$$
\bar{\mu}(I(\mathscr{L}))=\bar{\mu}^{*}\left(\bigcup_{n=1}^{\infty} V\left(A_{n}^{\prime}\right)\right) .
$$

Now the proof can be completed as in part (b)5 of Theorem 3.2.

Now, we turn our attention to the case $\mu \in J(\mathscr{L})$. To handle this case in a manner similar to the cases of $M_{\sigma}(\mathscr{L})$ and $M^{\sigma}(\mathscr{L})$, we must introduce the appropriate outer 
measures. We first note trivially that $\mu \in J(\mathscr{L})$ if and only if whenever $L_{n} \downarrow L, L_{n}, L \in \mathscr{L}$, then $\mu\left(L_{n} \cap L^{\prime}\right) \rightarrow 0$. Next, for each $L \in \mathscr{L}$, we consider the following outer measure. Consider the covering class consisting of $\varnothing$ and $\mathscr{L}^{\prime} \cup L$. Now, define for $E \subset \mathbf{X}$ and $\mu \in M(\mathscr{L})$

$$
\mu_{L}^{\prime \prime}(E)=\inf \left\{\sum_{i=1}^{\infty} \mu\left(L_{i}^{\prime} \cup L\right) \mid E \subset \bigcup_{i=1}^{\infty}\left(L_{i}^{\prime} \cup L\right), L_{i} \in \mathscr{L}\right\} .
$$

We note the following easily proved facts.

(1) $\mu_{L}^{\prime \prime}$ is an outer measure for each $L \in \mathscr{L}$.

(2) $\mu_{\phi}^{\prime \prime}=\mu^{\prime \prime}$.

(3) If $\mu \in I(\mathscr{L})$, then $\mu \leq \mu_{L}^{\prime \prime}$ on $\mathscr{L}$ for any $L \in \mathscr{L}$ if and only if $\mu \in \Phi(\mathscr{L})$.

(4) If $\mu \in M(\mathscr{L})$, then $\mu_{L}^{\prime \prime}(\mathbf{X})=\mu(\mathbf{X})$ for any $L \in \mathscr{L}$.

(5) For $E \subset \mathbf{X}, \mu_{L}^{\prime \prime}(E)=\bar{\mu}_{W(L)}^{\prime \prime}(E)$ and $\mu_{L}^{\prime \prime}(E)=\bar{\mu}_{V(L)}^{\prime \prime}(E)$.

Proof. We just show (3) and (4).

(3) Suppose that $\mu \in \Phi(\mathscr{L})$ and that there exists $A \in \mathscr{L}$ such that $\mu(A)=1$ and $\mu_{L}^{\prime \prime}(A)=0$ for some $L \in \mathscr{L}$. Then

$$
A \subset \bigcup_{i=1}^{\infty}\left(L_{i}^{\prime} \cup L\right) \quad \text { and } \quad \mu\left(L_{i}^{\prime} \cup L\right)=0 \text { for all } i .
$$

Therefore, $\mu\left(L_{i} \cap L^{\prime}\right)=1$ for all $i$. So, $\mu\left(L_{i}\right)=1$ for all $i$, and $\mu\left(L^{\prime}\right)=1$.

Now, $\bigcap_{n=1}^{\infty}\left(L_{i} \cap L^{\prime}\right) \cap A=\varnothing$, so that $\bigcap_{n=1}^{\infty} L_{i} \cap A \subset L$, and $\mu\left(L_{i} \cap A\right)=1$ for all $i$ and $\mu(L)=0$. This is a contradiction since $\mu \in \Phi(\mathscr{L})$. (Namely, suppose that $\mu \in \Phi(\mathscr{L})$ and suppose that $\bigcap_{i=1}^{\infty} A_{i} \subset B$ with $A_{i}, B \in \mathscr{L}$, and $\mu\left(A_{i}\right)=1$ for all $i$. Then $B^{\prime} \subset \bigcup_{i=1}^{\infty} A_{i}^{\prime}$, $\mu\left(A_{i}^{\prime}\right)=0$. Therefore, $\mu^{\prime \prime}\left(B^{\prime}\right)=0$, and so $\mu\left(B^{\prime}\right)=0$ since $\mu \in \Phi(\mathscr{L})$. Therefore, $\mu(B)=1$.) Conversely, suppose that $\mu \leq \mu_{L}^{\prime \prime}$ for all $L \in \mathscr{L}$. Then $\mu \leq \mu_{\phi}^{\prime \prime}=\mu^{\prime \prime}$ and so $\mu \in I_{\sigma}(\mathscr{L})$.

Suppose that $L_{n} \downarrow L, L_{n}, L \in \mathscr{L}$ and $\mu\left(L_{n}\right)=1$ for all $n$ and $\mu(L)=0$, so that $\mu\left(L^{\prime}\right)=1$. Then $L^{\prime}=\bigcup_{n=1}^{\infty} L_{n}^{\prime}, \mu\left(L_{n}^{\prime}\right)=0$. We have $\mathbf{X}=L^{\prime} \cup L=\left(\bigcup L_{n}^{\prime}\right) \cup L$ and $\mu\left(L_{n}^{\prime} \cup L\right)=0$. Therefore, $\mu_{L}^{\prime \prime}(\mathbf{X})=0$, a contradiction, since $1=\mu(\mathbf{X}) \leq \mu_{L}^{\prime \prime}(\mathbf{X})$.

Therefore, we must have $\mu \in \Phi(\mathscr{L})$.

(4) Clearly, $\mu_{L}^{\prime \prime}(\mathbf{X}) \leq \mu(\mathbf{X})$ for $L \in \mathscr{L}$. Suppose that $\mu_{L}^{\prime \prime}(\mathbf{X}) \leq \mu(\mathbf{X})$ for some $L \in \mathscr{L}$. Then there exists $L_{i} \in \mathscr{L}$ such that

$$
\mathbf{X} \subset \bigcup_{i=1}^{\infty}\left(L_{i}^{\prime} \cup L\right) \quad \text { and } \quad \sum_{i=1}^{\infty} \mu\left(L_{i}^{\prime} \cup L\right)<\mu(\mathbf{X}) .
$$

Therefore,

$$
\mu(\mathbf{X})>\sum_{i=1}^{\infty} \mu\left(L_{i}^{\prime} \cup L\right)=\lim _{n \rightarrow \infty} \sum_{i=1}^{n} \mu\left(L_{i}^{\prime} \cup L\right) \geq \lim _{n \rightarrow \infty} \mu\left(\bigcup_{i=1}^{n}\left(L_{i}^{\prime} \cup L\right)\right) .
$$

But, $\bigcup_{i=1}^{n}\left(L_{i}^{\prime} \cup L\right) \uparrow \mathbf{X}$ and so $\lim _{n \rightarrow \infty} \mu\left(\bigcup_{i=1}^{n}\left(L_{i}^{\prime} \cup L\right)\right)=\mu(\mathbf{X})$, a contradiction. Hence,

$$
\mu_{L}^{\prime \prime}(\mathbf{X})=\mu(\mathbf{X}) \text { for all } L \in \mathscr{L} .
$$


Once again, we have the option of considering the two Wallman pairs $I_{R}(\mathscr{L}), W(\mathscr{L})$ or $I(\mathscr{L}), V(\mathscr{L})$. We will just state and prove the result for the latter pair.

THEOREM 3.4. Let $\mathscr{L}$ be $T_{0}$ and $\mu \in M(\mathscr{L})$. Then

(1) If $\mu \in J(\mathscr{L})$, then

$$
\bar{\mu}_{V(L)}^{\prime \prime}(\mathbf{X})=\bar{\mu}_{V(L)}^{\prime \prime}\left(I^{\sigma}(\mathscr{L})\right)=\bar{\mu}_{V(L)}^{\prime \prime}\left(I_{\sigma}(\mathscr{L})\right)=\bar{\mu}(I(\mathscr{L})) \quad \text { for all } L \in \mathscr{L} .
$$

(2) If $\mu \in M(\mathscr{L})$ and if $\bar{\mu}_{V(L)}^{\prime \prime}$ is regular for all $L \in \mathscr{L}$, then if $\bar{\mu}_{V(L)}^{\prime \prime}(\mathbf{X})=\bar{\mu}(I(\mathscr{L}))$ for all $L \in \mathscr{L}$, or if $\bar{\mu}_{V(L)}^{\prime \prime}(\Phi(\mathscr{L}))=\bar{\mu}(I(\mathscr{L}))$ for all $L \in \mathscr{L}$ or if $\bar{\mu}_{V(L))}^{\prime \prime}\left(I^{\sigma}(\mathscr{L})\right)=\bar{\mu}(I(\mathscr{L}))$ for all $L \in \mathscr{L}$, then $\mu \in J(\mathscr{L})$.

(3) If $\mu \in I(\mathscr{L})$, then $\mu \in \Phi(\mathscr{L})$ if and only if $\bar{\mu}_{V(L)}^{\prime \prime}(\mathbf{X})=\bar{\mu}(I(\mathscr{L}))$ or $\bar{\mu}_{V(L)}^{\prime \prime}(\Phi(\mathscr{L}))=$ $\bar{\mu}(I(\mathscr{L}))$ or $\bar{\mu}_{V(L)}^{\prime \prime}\left(I^{\sigma}(\mathscr{L})\right)=\bar{\mu}(I(\mathscr{L}))$ for all $L \in \mathscr{L}$.

Proof. We prove (1) and a part of (2).

(1) Let $\mu \in J(\mathscr{L})$. Then $\bar{\mu} \in J(V(\mathscr{L})$ ). By properties (4) and (5) of the outer measure $\mu_{L}^{\prime \prime}$, we have

$$
\mu_{L}^{\prime \prime}(\mathbf{X})=\bar{\mu}_{V(L)}^{\prime \prime}(\mathbf{X})=\mu(\mathbf{X}) \quad \text { for all } L \in \mathscr{L}
$$

Since

$$
V(\mathbf{X})=I(\mathscr{L}), \quad \bar{\mu}(I(\mathscr{L}))=\bar{\mu}(V(\mathbf{X}))=\mu(\mathbf{X}) .
$$

Now, $\mathbf{X} \subset I^{\sigma}(\mathscr{L}) \subset I_{\sigma}(\mathscr{L}) \subset I(\mathscr{L})$ and so, by monotonicity of $\bar{\mu}_{V(L)}^{\prime \prime}$,

$$
\bar{\mu}_{V(L)}^{\prime \prime}(\mathbf{X}) \leq \bar{\mu}_{V(L)}^{\prime \prime}\left(I^{\sigma}(\mathscr{L})\right) \leq \bar{\mu}_{V(L)}^{\prime \prime}\left(I_{\sigma}(\mathscr{L})\right) \leq \bar{\mu}_{V(L)}^{\prime \prime}(I(\mathscr{L})) .
$$

By property $(4), \bar{\mu}_{V(L)}^{\prime \prime}(I(\mathscr{L}))=\bar{\mu}(I(\mathscr{L}))$. It follows from this and inequality (3.31) that

$$
\bar{\mu}(I(\mathscr{L}))=\bar{\mu}_{V(L)}^{\prime \prime}\left(I^{\sigma}(\mathscr{L})\right)=\bar{\mu}_{V(L)}^{\prime \prime}\left(I_{\sigma}(\mathscr{L})\right)=\bar{\mu}_{V(L)}^{\prime \prime}(\mathbf{X}) \quad \text { for any } L \in \mathscr{L} .
$$

(2) Let $\mu \in M(\mathscr{L})$. We show that if $\bar{\mu}_{V(L)}^{\prime \prime}$ is regular for all $L \in \mathscr{L}$ and if $\bar{\mu}_{V(L)}^{\prime \prime}(\mathbf{X})=$ $\bar{\mu}(I(\mathscr{L}))$, then $\mu \in J(\mathscr{L})$.

Let $L_{n} \in \mathscr{L}, n=1,2, \ldots, L \in \mathscr{L}$ such that $L_{n} \downarrow L$. Then $L_{n} \cap L^{\prime} \downarrow \varnothing$ and so in $I(\mathscr{L})$, $V\left(L_{n} \cap L^{\prime}\right) \downarrow$. Hence, $\bigcap_{n=1}^{\infty} V\left(L_{n} \cap L^{\prime}\right) \subset I(\mathscr{L})-\mathbf{X}$, so that $\mathbf{X} \subset \bigcup_{n=1}^{\infty} V\left(L_{n}^{\prime} \cup L\right)$.

It follows, by monotonicity of $\bar{\mu}_{V(L)}^{\prime \prime}$, that

$$
\bar{\mu}_{V(L)}^{\prime \prime}(\mathbf{X}) \leq \bar{\mu}_{V(L)}^{\prime \prime}\left(\bigcup_{n=1}^{\infty} V\left(L_{n}^{\prime} \cup L\right)\right) \leq \bar{\mu}_{V(L)}^{\prime \prime}(I(\mathscr{L})) .
$$

By hypothesis, $\bar{\mu}_{V(L)}^{\prime \prime}(\mathbf{X})=\bar{\mu}(I(\mathscr{L}))$; and by definition, $\bar{\mu}_{V(L)}^{\prime \prime}(I(\mathscr{L})) \leq \bar{\mu}(I(\mathscr{L}))$. The preceding argument shows that

$$
\bar{\mu}(I(\mathscr{L}))=\bar{\mu}_{V(L)}^{\prime \prime}\left(\bigcup_{n=1}^{\infty} V\left(L_{n}^{\prime} \cup L\right)\right)=\lim _{n \rightarrow \infty} \bar{\mu}_{V(L)}^{\prime \prime}\left(V\left(L_{n}^{\prime} \cup L\right)\right)
$$

by regularity of $\bar{\mu}_{V(L)}^{\prime \prime}$. But, $\mu(\mathbf{X})=\bar{\mu}(I(\mathscr{L}))$ and we have

$$
\mu(\mathbf{X})=\lim _{n \rightarrow \infty} \bar{\mu}_{V(L)}^{\prime \prime}\left(V\left(L_{n}^{\prime} \cup L\right)\right) .
$$


For all $n$,

$$
\bar{\mu}_{V(L)}^{\prime \prime}\left(V\left(L_{n}^{\prime} \cup L\right)\right) \leq \bar{\mu}\left(V\left(L_{n}^{\prime} \cup L\right)\right)=\mu\left(L_{n}^{\prime} \cup L\right) \leq \mu(\mathbf{X})
$$

since $L_{n}^{\prime} \cup L \uparrow \mathbf{X}$. Hence, $\mu(\mathbf{X})=\lim _{n \rightarrow \infty} \mu\left(L_{n}^{\prime} \cup L\right)$. It follows from this that $\lim _{n \rightarrow \infty} \mu\left(L_{n} \cap\right.$ $\left.L^{\prime}\right)=0$, whenever, $L_{n} \downarrow L$ in $\mathscr{L}$. Therefore, $\mu \in M(\mathscr{L})$.

4. Measurability and smoothness. In this section, we wish to show how the measurability of certain sets in the Wallman spaces affect the smoothness of the given measure in the original space. To be more specific, we first introduce the following theorem.

THEOREM 4.1. Let $\mathbf{X}$ be an arbitrary nonempty set and $\mathscr{L}$ a $T_{0}$ lattice of subsets containing $\varnothing, \mathbf{X}$.

(a) Let $\mu \in M(\mathscr{L})$. Suppose that $\mathrm{X} \in S_{\bar{\mu}^{\prime}}$. Then $\mathrm{X} \in S_{\bar{\mu}^{\prime \prime}}$ and if $\bar{\mu}^{\prime \prime}$ is regular, then $\mu \in M_{\sigma}(\mathscr{L})$.

(b) Let $\mu \in M(\mathscr{L})$. If $I_{\sigma}(\mathscr{L}) \in S_{\bar{\mu}}$, then $I_{\sigma}(\mathscr{L}) \in S_{\bar{\mu}^{\prime \prime}}$ and if $\bar{\mu}^{\prime \prime}$ is regular, then $\mu \in$ $M_{\sigma}(\mathscr{L})$.

Proof. We prove (a) only since the proof of (b) is similar.

(a) Let $\mu \in M(\mathscr{L})$. Since $V(\mathscr{L})$ is compact, $\bar{\mu} \in M_{\sigma}(V(\mathscr{L}))$. Since $\mathbf{X} \in S_{\bar{\mu}^{\prime}}$,

$$
\bar{\mu}(I(\mathscr{L}))=\bar{\mu}^{\prime}(\mathbf{X})+\bar{\mu}^{\prime}(I(\mathscr{L})-\mathbf{X}) .
$$

Now, $\bar{\mu}(I(\mathscr{L}))=\mu(\mathbf{X})=\bar{\mu}^{\prime}(\mathbf{X})$, and so the preceding equation gives $\bar{\mu}^{\prime}(I(\mathscr{L})-\mathbf{X})=0$. Since $\bar{\mu}^{\prime \prime} \leq \bar{\mu}^{\prime}$, we have $\bar{\mu}^{\prime \prime}(I(\mathscr{L})-\mathbf{X})=0$. Therefore, $I(\mathscr{L})-\mathbf{X} \in S_{\bar{\mu}^{\prime \prime}}$ and so $\mathbf{X} \in S_{\bar{\mu}^{\prime \prime}}$. And we must have $\bar{\mu}^{\prime \prime}(\mathbf{X})=\bar{\mu}(I(\mathscr{L}))$.

In addition, if $\bar{\mu}^{\prime \prime}$ is regular, then the preceding argument, together with Remark 1, shows that $\mu \in M_{\sigma}(\mathscr{L})$.

REMARK 2. Instead of considering the pair $I(\mathscr{L}), V(\mathscr{L})$ as in Theorem 4.1, we could consider $I_{R}(\mathscr{L}), W(\mathscr{L})$, assuming that $\mathscr{L}$ is $T_{0}$ and disjunctive, to obtain statements similar to (a) and (b) above. For example, in this case, if $\mu \in M(\mathscr{L})$ and if $I_{R}^{\sigma}(\mathscr{L}) \in S_{\bar{\mu}^{\prime}}$ then $I_{R}^{\sigma}(\mathscr{L}) \in S_{\bar{\mu}^{\prime \prime}}$ and if $\bar{\mu}^{\prime \prime}$ is regular, then $\mu \in M_{\sigma}(\mathscr{L})$.

We now investigate measurability and $\sigma$-smoothness on $\mathbf{A}(\mathscr{L})$, that is, with the assumption of countable additivity.

THEOREM 4.2. Let $\mathrm{X}$ be an arbitrary nonempty set and $\mathscr{L}$ a $T_{0}$ disjunctive lattice of subsets of $\mathbf{X}$ such that $\varnothing, \mathbf{X} \in \mathscr{L}$. Let $\mu \in M_{R}(\mathscr{L})$ (then $\bar{\mu} \in M_{R}^{\sigma}(W(\mathscr{L}))$ ).

(a) If $\mathbf{X} \in S_{\bar{\mu}^{*}}$, then $\mathbf{X} \in S_{\bar{\mu}^{*}}$ and $\mu \in M_{R}^{\sigma}(\mathscr{L})$.

(b) If $I_{R}^{\sigma}(\mathscr{L}) \in S_{\bar{\mu}^{\bullet}}$, then $I_{R}^{\sigma}(\mathscr{L}) \in S_{\bar{\mu}^{*}}$ and $\mu \in M_{R}^{\sigma}(\mathscr{L})$.

Proof. The proofs are quite similar so we just prove (a).

(a) Let $\mu \in M_{R}(\mathscr{L})$. Then $\bar{\mu} \in M_{R}(W(\mathscr{L}))$. Since $W(\mathscr{L})$ is countably compact, $\bar{\mu} \in$ $M_{\sigma}(W(\mathscr{L}))$, whence, $\bar{\mu} \in M_{R}^{\sigma}(W(\mathscr{L}))$, and so $\bar{\mu}^{*}$ is regular.

Since $\mathbf{X} \in S_{\bar{\mu} \cdot}$,

$$
\bar{\mu} \bullet I_{R}(\mathscr{L})=\bar{\mu} \bullet(\mathbf{X})+\bar{\mu} \bullet\left(I_{R}(\mathscr{L})-\mathbf{X}\right) .
$$


Now, $\bar{\mu} \bullet(W(\mathbf{X}))=\bar{\mu} \bullet\left(I_{R}(\mathscr{L})\right)$ and so $\bar{\mu} \bullet(X)=\bar{\mu} \bullet\left(I_{R}(\mathscr{L})\right)$. It follows from the preceding argument that

$$
\bar{\mu}^{\bullet}(\mathbf{X})=\bar{\mu} \cdot(\mathbf{X})+\bar{\mu}^{\bullet}\left(I_{R}(\mathscr{L})-\mathbf{X}\right) .
$$

Therefore,

$$
\bar{\mu} \cdot\left(I_{R}(\mathscr{L})-X\right)=0 .
$$

Since $\bar{\mu}^{*} \leq \bar{\mu}^{\bullet}$, (4.4) shows that $\bar{\mu}^{*}\left(I_{R}(\mathscr{L})-\mathbf{X}\right)=0$. Therefore, $I_{R}(\mathscr{L})-\mathbf{X} \in S_{\bar{\mu}^{*}}$ and so $\mathbf{X} \in S_{\bar{\mu}^{*}}$. Now, $\bar{\mu} \in M^{\sigma}(W(\mathscr{L}))$. So, $\bar{\mu}\left(I_{R}(\mathscr{L})\right)=\bar{\mu}^{*}\left(I_{R}(\mathscr{L})\right)$.

Since $\mathbf{X} \in S_{\bar{\mu}^{*}}$,

$$
\bar{\mu}^{*}\left(I_{R}(\mathscr{L})\right)=\bar{\mu}^{*}(\mathbf{X})+\bar{\mu}^{*}\left(I_{R}(\mathscr{L})-\mathbf{X}\right)
$$

and, by (4.4),

$$
\bar{\mu}^{*}\left(I_{R}(\mathscr{L})\right)=\bar{\mu}^{*}(\mathbf{X})=\bar{\mu}\left(I_{R}(\mathscr{L})\right) .
$$

By (4.6) and Remark 2, $\mu \in M^{\sigma}(\mathscr{L})$.

The hypothesis $\mu \in M_{R}(\mathscr{L})$ shows that $\mu \in M_{R}^{\sigma}(\mathscr{L})$.

REMARK 3. If, instead of the Wallman pair $I_{R}(\mathscr{L}), W(\mathscr{L})$, we consider $I(\mathscr{L}), V(\mathscr{L})$ with just the assumption that $\mathscr{L}$ is $T_{0}$, we get similarly

If $\mu \in M_{R}(\mathscr{L})$, then

(a) If $\mathbf{X} \in S_{\bar{\mu}^{*}}$, then $\mathbf{X} \in S_{\bar{\mu}^{*}}$, and $\mu \in M_{R}^{\sigma}(\mathscr{L})$.

(b) If $I^{\sigma}(\mathscr{L}) \in S_{\bar{\mu}^{\bullet}}$, then $I^{\sigma}(\mathscr{L}) \in S_{\bar{\mu}^{*}}$, and $\mu \in M_{R}^{\sigma}(\mathscr{L})$.

If we do not assume that $\mu \in M_{R}(\mathscr{L})$ in either case but only that $\mu \in M(\mathscr{L})$, the problem of then determining in terms of measurable sets that $\mu \in M^{\sigma}(\mathscr{L})$ becomes much more difficult. This is because $\mu \in M(\mathscr{L})$ only implies that $\bar{\mu} \in M_{\sigma}\left(W(\mathscr{L})\right.$ ) or $M_{\sigma}(V(\mathscr{L}))$ depending on the Wallman space considered, and not a priori, as in the regular case that $\bar{\mu}$ is countably additive. We can, however, prove the following theorem.

THEOREM 4.3. Let $\mathrm{X}$ be an arbitrary nonempty set and $\mathscr{L}$ a $T_{0}$ lattice of subsets of $\mathrm{X}$ such that $\varnothing, \mathrm{X} \in \mathscr{L}$. Let $\mu \in M(\mathscr{L})$. If $I^{\sigma}(\mathscr{L}) \in S_{\bar{\mu}^{\prime}}$ then $I^{\sigma}(\mathscr{L}) \in S_{\bar{\mu}^{*}}$ and $\mu \in M^{\sigma}(\mathscr{L})$ if $\bar{\mu}^{*}$ is regular and if $\bar{\mu}^{*}\left(I^{\sigma}(\mathscr{L})\right)=\bar{\mu}^{\prime \prime}\left(I^{\sigma}(\mathscr{L})\right)$.

Proof. Let $\mu \in M(\mathscr{L})$. Since $V(\mathscr{L})$ is countably compact, $\bar{\mu} \in M_{\sigma}(V(\mathscr{L})$ ), which implies that $\bar{\mu}^{\prime \prime}(I(\mathscr{L}))=\bar{\mu}(I(\mathscr{L}))$. We always have

$$
\bar{\mu}(I(\mathscr{L}))=\bar{\mu}^{\prime}(I(\mathscr{L})) .
$$

Since $I^{\sigma}(\mathscr{L}) \in S_{\bar{\mu}^{\prime}}$,

$$
\bar{\mu}^{\prime}(I(\mathscr{L}))=\bar{\mu}^{\prime}\left(I^{\sigma}(\mathscr{L})\right)+\bar{\mu}^{\prime}\left(I(\mathscr{L})-I^{\sigma}(\mathscr{L})\right) .
$$

Therefore, by (4.7),

$$
\bar{\mu}(I(\mathscr{L}))=\bar{\mu}^{\prime}\left(I^{\sigma}(\mathscr{L})\right)+\bar{\mu}^{\prime}\left(I(\mathscr{L})-I^{\sigma}(\mathscr{L})\right) .
$$

But

$$
\bar{\mu}(I(\mathscr{L}))=\bar{\mu}(V(\mathbf{X}))=\mu(\mathbf{X}) .
$$


Hence, by (4.9) and (4.10),

$$
\mu(\mathbf{X})=\bar{\mu}^{\prime}\left(I^{\sigma}(\mathscr{L})\right)+\bar{\mu}^{\prime}\left(I(\mathscr{L})-I^{\sigma}(\mathscr{L})\right) .
$$

Now, $\mu(\mathbf{X}) \leq \bar{\mu}^{\prime}(\mathbf{X}) \leq \bar{\mu}^{\prime}\left(I^{\sigma}(\mathscr{L})\right)$, and so by (4.10)

$$
\mu(\mathbf{X}) \geq \mu(\mathbf{X})+\bar{\mu}^{\prime}\left(I(\mathscr{L})-I^{\sigma}(\mathscr{L})\right) .
$$

So, $0 \leq \bar{\mu}^{\prime}\left(I(\mathscr{L})-I^{\sigma}(\mathscr{L})\right) \leq 0$.

Therefore, $\bar{\mu}^{\prime}\left(I(\mathscr{L})-I^{\sigma}(\mathscr{L})\right)=0$. Since $\bar{\mu}^{*} \leq \bar{\mu}^{\prime \prime} \leq \bar{\mu}^{\prime}$,

$$
\mu^{*}\left(I(\mathscr{L})-I^{\sigma}(\mathscr{L})\right)=0 .
$$

Therefore, $I(\mathscr{L})-I^{\sigma}(\mathscr{L}) \in S_{\bar{\mu}^{*}}$ and so $I^{\sigma}(\mathscr{L}) \in S_{\bar{\mu}^{*}}$.

It follows that

$$
\bar{\mu}^{*}(I(\mathscr{L}))=\bar{\mu}^{*} I^{\sigma}(\mathscr{L})+\bar{\mu}^{*}\left(I(\mathscr{L})-I^{\sigma}(\mathscr{L})\right) .
$$

Thus,

$$
\bar{\mu}^{*}(I(\mathscr{L}))=\bar{\mu}^{*}\left(I^{\sigma}(\mathscr{L})\right) .
$$

Now,

$$
\bar{\mu}(I(\mathscr{L})) \leq \bar{\mu}^{\prime \prime}(I(\mathscr{L})) \leq \bar{\mu}^{\prime}(I(\mathscr{L})) .
$$

By (4.7),

$$
\bar{\mu}(I(\mathscr{L}))=\bar{\mu}^{\prime \prime}(I(\mathscr{L})) .
$$

We have $\bar{\mu}^{*}(I(\mathscr{L})) \leq \bar{\mu}(I(\mathscr{L}))$ and so

$$
\bar{\mu}^{*}(I(\mathscr{L})) \leq \bar{\mu}^{\prime \prime}(I(\mathscr{L})) .
$$

By (4.17),

$$
\bar{\mu}^{*}(I(\mathscr{L}))=\bar{\mu}^{\prime \prime}(I(\mathscr{L})) .
$$

By hypothesis and (4.15),

$$
\bar{\mu}^{\prime \prime}\left(I^{\sigma}(\mathscr{L})\right)=\bar{\mu}^{*}\left(I^{\sigma}(\mathscr{L})\right)=\bar{\mu}^{*}(I(\mathscr{L})) \leq \bar{\mu}(I(\mathscr{L}))=\bar{\mu}^{\prime \prime}(I(\mathscr{L})) .
$$

Therefore, the inequality shows that $\bar{\mu}(I(\mathscr{L}))=\bar{\mu}^{*}\left(I^{\sigma}(\mathscr{L})\right)$. By Theorem 3.3(2), $\mu \in$ $M^{\sigma}(\mathscr{L})$.

ACKNOWLEDGEMENT. The author wishes to thank the referees for several useful suggestions made to enhance the clarity of the paper.

\section{REFERENCES}

[1] J. A. Allan, Topological properties of generalized Wallman spaces and lattice relations, Internat. J. Math. Math. Sci. 19 (1996), no. 4, 717-722. CMP 96 15. Zbl 864.28005. 
[2] G. Bachman and P. Stratigos, Criteria for $\sigma$-smoothness, $\tau$-smoothness, and tightness of lattice regular measures, with applications, Canad. J. Math. 33 (1981), no. 6, 14981525. MR 83c:28010. Zbl 474.28006.

[3] G. Bachman and M. Szeto, On strongly measure replete lattices and the general Wallman remainder, Fund. Math. 122 (1984), no. 3, 199-217. MR 86d:28012. Zbl 567.28006.

[4] J. Camacho, Jr., On topological properties of certain Wallman spaces, J. Math. Sci. (Calcutta) 7 (1996), no. 1, 33-44. MR 98c:28013.

[5] M. C. Figueres, Lattice separation, coseparation and regular measures, Internat. J. Math. Math. Sci. 19 (1996), no. 4, 773-779. MR 97f:28036. Zbl 857.28011.

[6] P. M. Grassi, Outer measures and associated lattice properties, Internat. J. Math. Math. Sci. 16 (1993), no. 4, 687-694. MR 95f:28003. Zbl 778.28009.

[7] M. E. Munroe, Introduction to measure and integration, Addison-Wesley Publishing Company, Cambridge, 1953. MR 14,734a. Zbl 050.05603.

[8] J. Ponnley, Outer measures, measurability, and lattice regular measures, Internat. J. Math. Math. Sci. 19 (1996), no. 2, 343-350. MR 97b:28017. Zbl 841.28004.

[9] M. Szeto, On normal lattices and separation properties of lattices, J. Indian Math. Soc. (N.S.) 58 (1992), no. 1-4, 51-64. MR 94h:06009. Zbl 883.06005.

[10] C. Traina, Outer measures associated with lattice measures and their application, Internat. J. Math. Math. Sci. 18 (1995), no. 4, 725-734. MR 96g:28019. Zbl 833.28006.

[11] _ On some properties and applications of general Wallman spaces, J. Math. Sci. (Calcutta) 7 (1996), no. 1, 1-16. MR 98c:28016.

Traina: St. John's UniVersity, DePARTMENT OF MATHEMATICS AND COMPUTER SCIENCE, 8000 UTOPIA PARKWAY, JAMAICA, NY 11439 


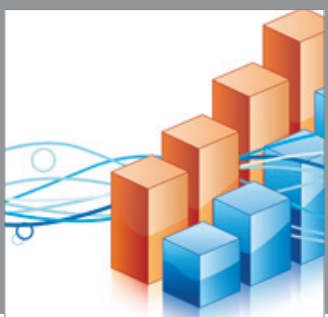

Advances in

Operations Research

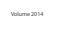

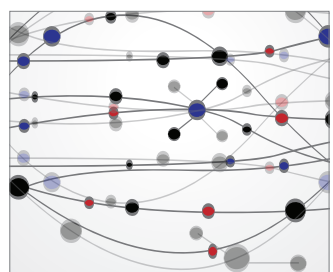

\section{The Scientific} World Journal
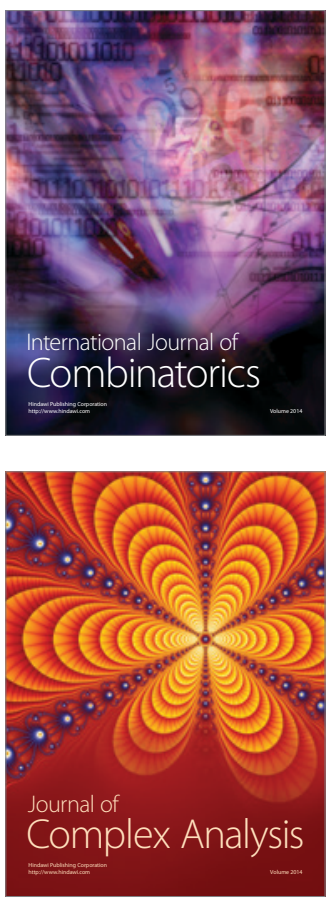

International Journal of

Mathematics and

Mathematical

Sciences
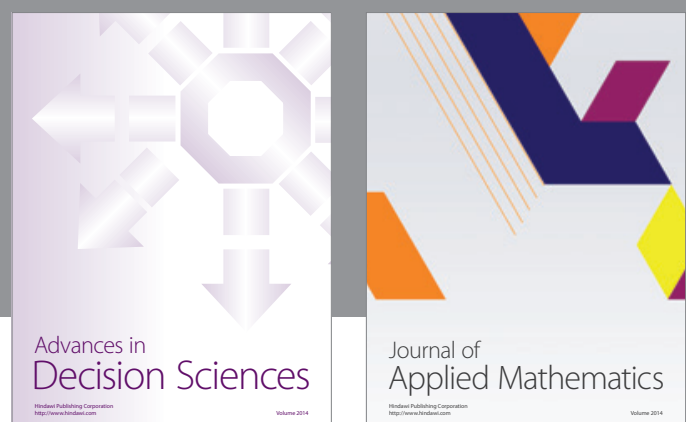

Journal of

Applied Mathematics
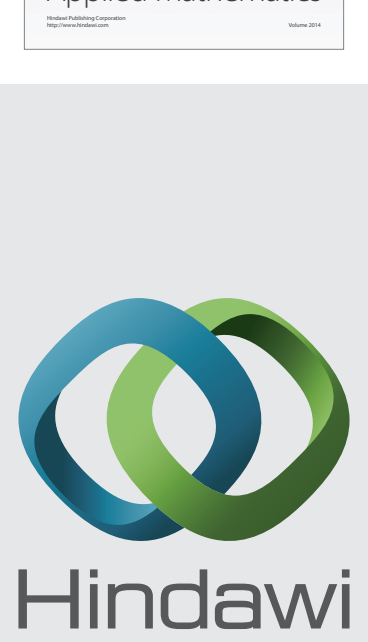

Submit your manuscripts at http://www.hindawi.com
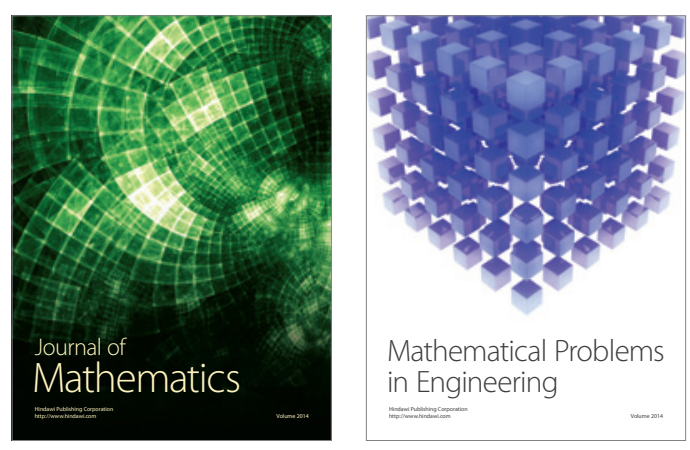

Mathematical Problems in Engineering
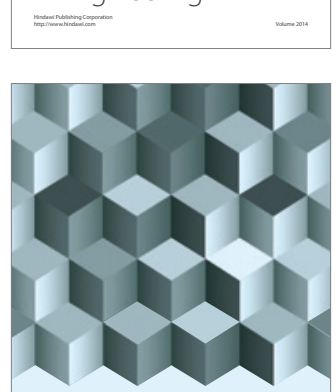

Journal of

Function Spaces
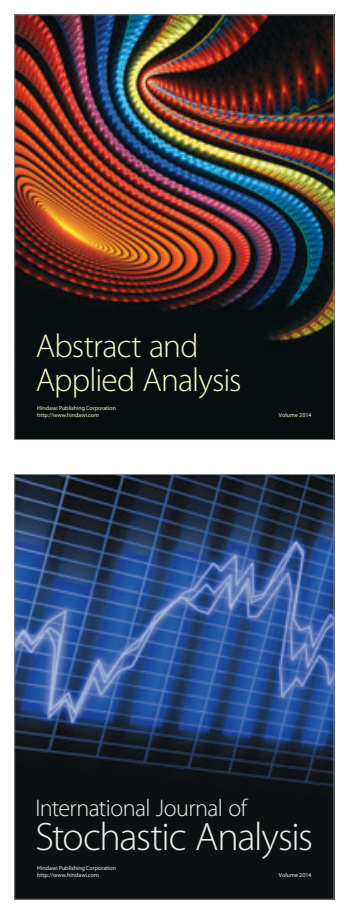

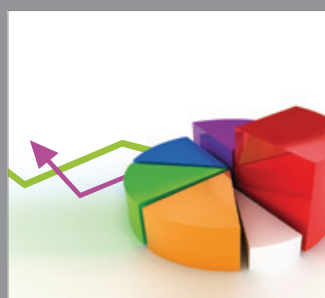

ournal of

Probability and Statistics

Promensencen
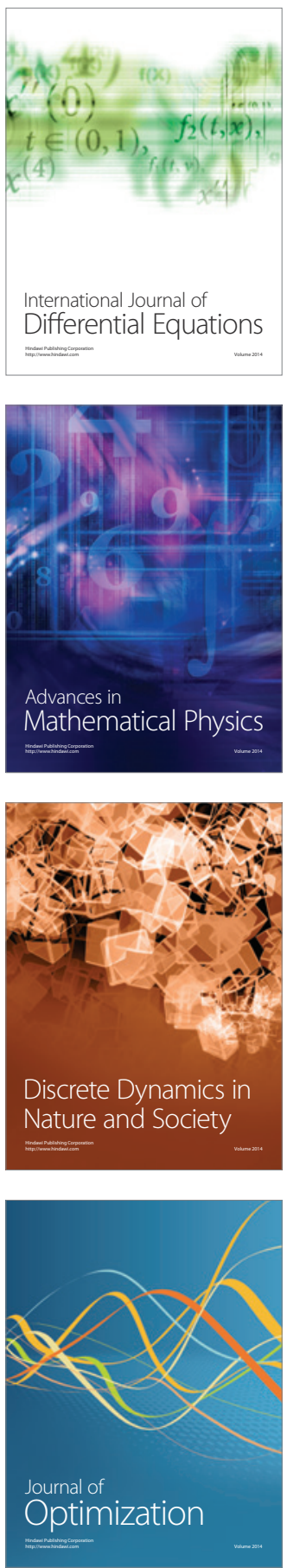